\title{
Dynamical refinement of modulated structures against electron diffraction data
}

Lukas Palatinus $^{1}$

${ }^{1}$ Department Of Structure Analysis, Institute Of Physics Of The CAS, Prague, Czech Republic E-mail: palat@fzu.cz

Refinement of crystal structures from electron diffraction data has long been hampered by the difficulties to appropriately model the diffracted intensities. Recently, the problem as overcome to a large extent by the development of the so-caled dynamical refinement method [1,2], where the model intensities are calculated using the dynamical diffraction theory. Because the diffraction patterns need to be calculated in arbitrary orientations of the crystal, Bloch-wave formalism was adopted for the calculation of the dynamical intensities as the most general approach. This approach is based on the Bloch theorem, which in turn is based on the assumption of the periodicity of the crystal structure.

This approach thus apparently excludes the application of the method to modulated structures. However, the Bloch-wave method can be generalized to modulated structures under the - generally valid - assumption that the structure factor amplitudes go to zero for higher-order satellite reflections. To arrive at this result, we may imagine approximating the modulated structure with a supercell. Although formally an incommensurately modulated structure cannot be approximated with a commensurate supercell, it is always possible to find a supercell approximation that generates reciprocal-lattice nodes which coincide with the satellite reflections within the experimental accuracy. If the structure factors of satellites above certain finite order are assumed to be zero, then it can be shown that the structure matrix, which needs to be diagonalized in the procedure of calculating the diffracted intensities, can be constructed as a band diagonal matrix with the half-width of the band given by the number of non-zero diffracted beams included in the building of the structure matrix. Such a matrix would still be extremely large, but the contribution of the high-order terms becomes progressively smaller and smaller, and thus the matrix can be cut to a much smaller matrix without significant loss of accuracy of the calculated intensities.

The approach has been incorporated in the crystallographic computing system Jana2006 and its feasibility and accuracy will be demonstrated on examples of inorganic incommensurately modulated structures.

[1] Palatinus, L. et al. (2015). Acta Cryst. A, 71, 235-244

[2] Palatinus, L. et al. (2015). Acta Cryst. B, 71, 740-751

Keywords: dynamical diffraction, incommensurately modulated structure, structure refinement 